\title{
Intensity-modulated carbon-ion radiation therapy versus intensity-modulated photon-based radiation therapy in locally recurrent nasopharyngeal carcinoma: a dosimetric comparison
}

This article was published in the following Dove Press journal: Cancer Management and Research

\author{
Lei Wang ${ }^{1-3}$ \\ Jiyi $\mathrm{Hu}^{2,4}$ \\ Xiaoli Liu ${ }^{2,5}$ \\ Weiwei Wang ${ }^{2,5}$ \\ Lin Kong ${ }^{1,2}$ \\ Jiade $\mathrm{J} \mathrm{Lu}^{2,4}$ \\ 'Department of Radiation Oncology, \\ Shanghai Proton and Heavy lon Center, \\ Fudan University Cancer Hospital, \\ Shanghai 201315, People's Republic of \\ China; ${ }^{2}$ Shanghai Engineering Research \\ Center of Proton and Heavy lon \\ Radiation Therapy, Shanghai, People's \\ Republic of China; ${ }^{3}$ Department of \\ Radiation Oncology, Longhua Hospital, \\ Shanghai University of Traditional \\ Chinese Medicine, Shanghai, People's \\ Republic of China; ${ }^{4}$ Department of \\ Radiation Oncology, Shanghai Proton and \\ Heavy Ion Center, Shanghai 201315, \\ People's Republic of China; ${ }^{5}$ Department \\ of Medical Physics, Shanghai Proton and \\ Heavy lon Center, Shanghai 201315, \\ People's Republic of China
}

Correspondence: Lin Kong Department of Radiation Oncology, Shanghai Proton and Heavy lon Center, Fudan University Cancer Hospital, 4365 Kangxin Road, Pudong, Shanghai 201321, People's Republic of China

Email lin.kong@sphic.org.cn

Jiade J Lu

Department of Radiation Oncology,

Shanghai Proton and Heavy lon Center,

4365 Kangxin Road, Pudong, Shanghai

20132I, People's Republic of China

Tel +862138296505

Email jiade.lu@sphic.org.cn
Purpose: To identify the specific dose advantage of intensity-modulated carbon-ion radiation therapy (IMCT) over photon-based intensity-modulated radiation therapy (IMRT) in the treatment of locally recurrent nasopharyngeal carcinoma (NPC).

Materials and methods: Ten patients with locally recurrent NPC underwent IMCT and IMRT planning. Target definition followed the recommendations of the International Commission on Radiation Units and Measurements (ICRU) reports no. 50, 62 and 83 . The real treatment plans which were delivered to patients were designed on the Siemens Syngo planning system while the control plans for dosimetric comparison were generated from the Varian Medical Systems. The optimization constraints of the two designs were basically the same. Target coverage was evaluated using the following parameters: Dmin, Dmax, D1, D2, D50, D95, D98 and D99. Target dose distribution and conformality were evaluated using the homogeneity index and conformity index. Normal tissue sparing of organs at risk (OARs) were evaluated using Dmean, D1 and Dmax. SPSS 22.0 software was used for data analysis.

Results: Both IMCT and IMRT plans met clinical prescription dose requirements. Target coverage of D1, D2, D50, D95, D98, D99 were not significantly different between the two plans $(P>0.05)$. The two plans showed satisfactory coverage of the target without significant difference. There was no significant difference in terms of the homogeneity and conformability between the two plans. Dosimetric parameters for the brain stem, spinal cord, parotid gland, optic chiasm, eyeball, lens, temporal lobe and inner ear were significantly reduced in the IMCT plan $(P<0.05)$.

Conclusion: As compared with photon-based IMRT, IMCT significantly reduces radiation dose to the OARs in the treatment of locally recurrent NPC while maintaining the dose coverage to the target volumes. Such a feature is particularly important for patients who experienced previous high-dose irradiation.

Keywords: locally recurrent nasopharyngeal carcinoma, carbon ion, dosimetry comparison

\section{Introduction}

Nasopharyngeal carcinoma (NPC) is one of the most commonly diagnosed head and neck malignancies in Southern China and Southeast Asia. Radiation therapy (RT) is the only definitive treatment modality for non-metastatic NPC. ${ }^{1}$ Despite its 
high efficacy, approximately $10 \%$ of patients suffer local recurrence after radical radiotherapy with curative intent. ${ }^{2}$

There are only few effective treatment modalities for the management of recurrent NPC, and each with substantial limitations. Surgical resection is highly effective only in small recurrent lesions in the post-nasal space and neck lymph nodes. ${ }^{3,4}$ Re-irradiation (Re-RT) remains the mainstay treatment for locoregional recurrence of NPC, and photon-based intensity-modulated radiation therapy (IMRT) is the most commonly used method. However, reported overall survival rateafter re-RT with IMRT is dismal and ranged between $30 \%$ and $60 \%{ }^{5-8}$ Most patients suffered from severe radiation-induced toxicities of the organs at risk (OARs) including mucosal ulceration/ necrosis, massive hemorrhage, and brain necrosis secondary to repeated high-dose irradiation.

Charged particle beams exhibit unique physical properties with sharp boundaries and Bragg peak effects. The beams of particle radiation therapy (PRT) deposit the majority of their energy within a short range to form a narrow and pointed Bragg peak. The dose is then rapidly attenuated after the Bragg peak. Such physical property confers PRT higher precision than photon-based radiotherapy for dose distribution. As such, particle beams are superior to photon beam in protecting normal tissues around tumors, especially in patients who have received high-dose radiotherapy previously. Carbon-ion beam (CIB) is further featured with a higher linear energy transfer (LET) and a higher relative biological effectiveness (RBE). Such property is important for cancer cells irradiated with high-dose photon radiation but recurred. Both the physical and biological characteristics make CIB potentially a more effective modality for locally recurrent cancer after previous X-ray radiation. The safety of proton and carbon-ion radiotherapy for OARs in the head and neck, including the optic nerve, optic chiasm, brain, spinal cord and brain stem has also been previously reported..$^{9-14}$

This aim of the current study is to compare and determine a better re-RT technique for locally recurrent NPC by undertaking dosimetric comparisons of treatment plans using intensity-modulated carbon-ion-based radiotherapy (IMCT) using pencil beam scanning technology and intensity-modulated photon-based RT (IMRT).

\section{Materials and methods}

\section{Patients and image acquisition}

Between August 2016 and March 2017, 10 consecutive patients with locally recurrent NPC underwent IMCT at the Shanghai Proton and Heavy Ion Center (SPHIC). All patients were also enrolled in a dose-escalating Phase I/II clinical trial at SPHIC. All patients provided written informed consent for the clinical trial and using their data for research. ${ }^{15}$ All patients were irradiated with IMRT for definitive purpose for non-metastatic NPC previously, and recurred at least 1 year after the completion of IMRT. Pathologic confirmations of local recurrence were obtained and distant metastases at the time of re-RT were ruled out using imaging studies including PET-CT for all 10 patients. Patients were immobilized in the supine position using a head and neck-shoulder thermoplastic mask. Computed tomography (CT) of the head and neck taken from $1.5 \mathrm{~cm}$ superior to the cranium to $2.0 \mathrm{~cm}$ inferior to the subclavian was taken for planning without contrast at $1.5-\mathrm{mm}$ slide thickness. Magnetic resonance imaging (MRI) under the same position with fixation was obtained for tumor localization and fusion for radiation planning prior to registration. The CT scans were transferred to the treatment planning system (TPS) and integrated with the MRI scan images for target delineation.

\section{Volume definition}

Target definition was in accordance with the International Commission on Radiation Units andMeasurements (ICRU) reports Nos. 50, 62 and 83. ${ }^{16}$ Gross tumor volume (GTV) was defined mainly by enhanced MRI with information from physical examination and PET-CT imaging. Clinical target volume (CTV) was defined as including $5 \mathrm{~mm}$ beyond the GTV for microscopic extension which was limited to as little as $1 \mathrm{~mm}$ near OARs. Planning target volume (PTV) was defined as a boundary of 3-6 $\mathrm{mm}$ placed by CTV to eliminate setup errors and uncertainty of dose distribution.

The OARs contoured on the planning CT included bilateral eyes, lens, optic nerves and chiasm, temporal lobes, temporomandibular joint (TMJ), parotid glands, inner ears, brain stem, and spinal cord. The contouring of the OARs followed the recommendations from Sun et al. ${ }^{17}$ All OARs were delineated by the attending radiation oncologist of the particular patient and reviewed by two other radiation oncologists specialized in head and neck oncology. Figure 1 shows OARs contoured on CT with MRI image fusion.

\section{Treatment plans}

A total of 20 plans (10 for IMCT and 10 for IMRT) were generalized and analyzed. The treatment plans for IMCT was generated using the Syngo ${ }^{\circledR}$ Particle Treatment Planning System (TPS) of Siemens (versions VC11 and 
A

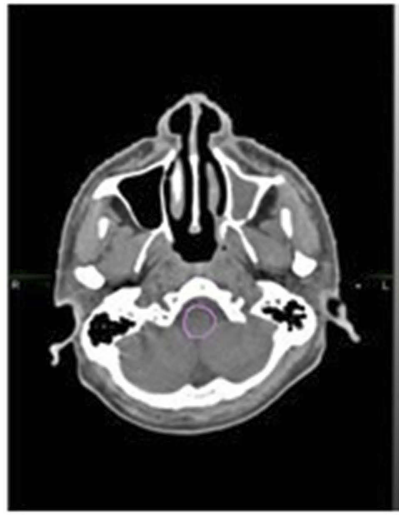

C

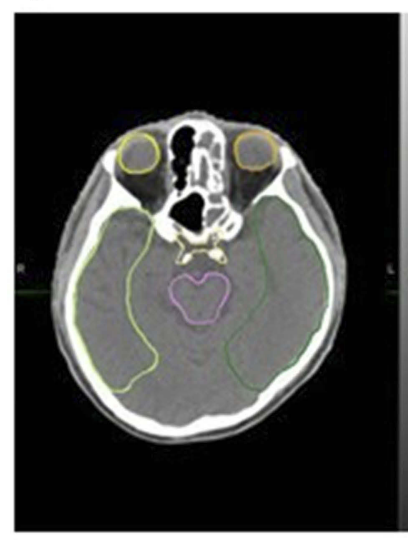

E

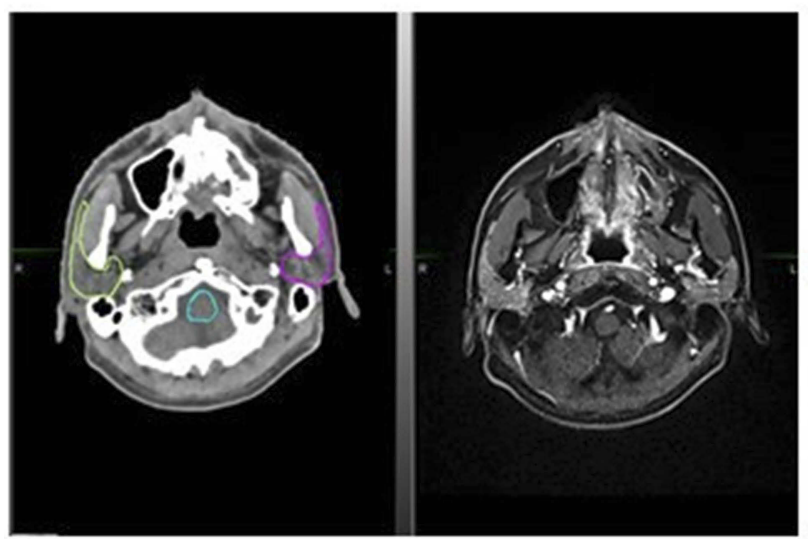

B

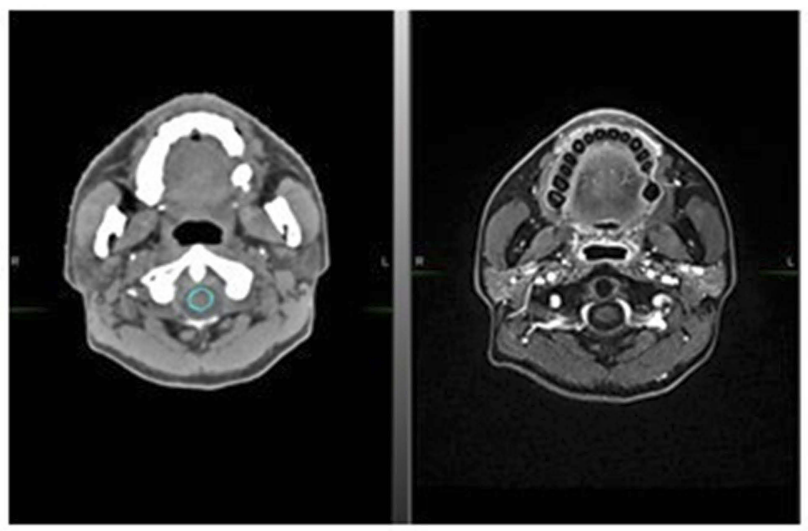

D

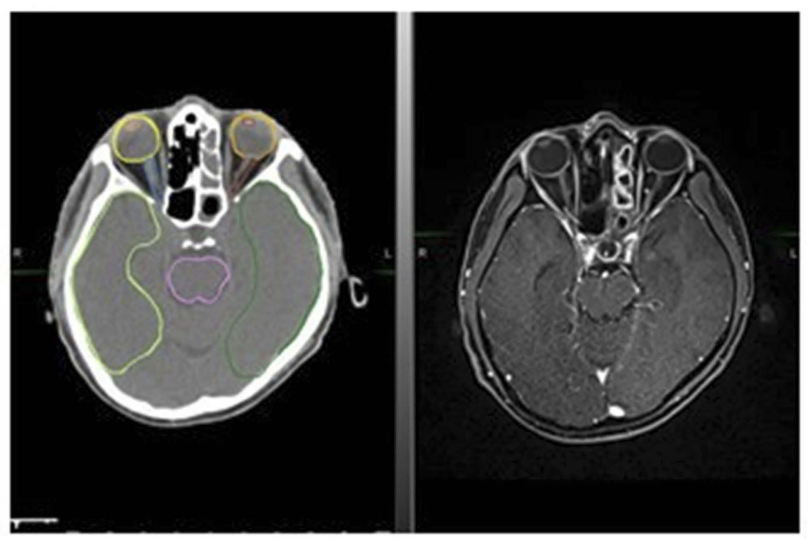

$\mathbf{F}$

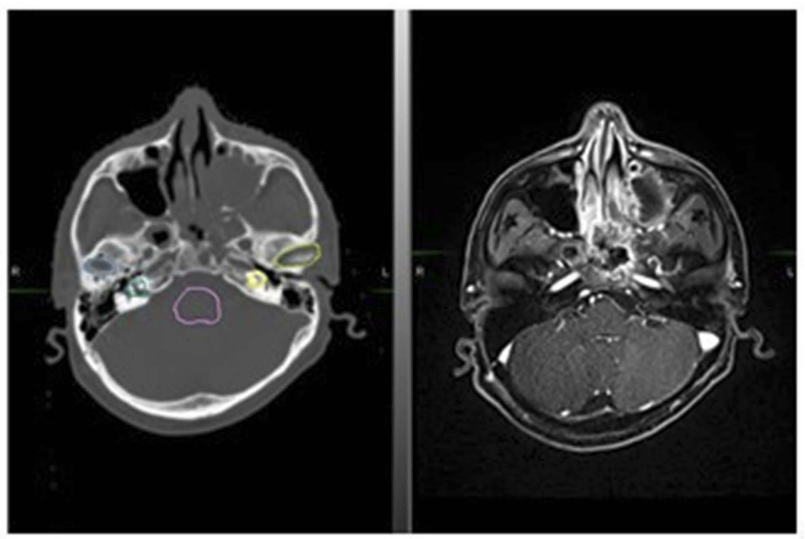

Figure I Organs at risk (OARs) contoured on planning computed tomography (CT) with magnetic resonance imaging (MRI) fusion. (A) Brain stem contoured on CT with MRI image fusion; (B) spinal cord contoured on CT with MRI image fusion; (C) eyeball, optic chiasm and temporal lobe contoured on CT with MRI image fusion; (D) lens and optic never contoured on CT with MRI image fusion; (E) parotid gland contoured on CT with MRI image fusion; and (F) temporomandibular joint and inner ear contoured on CT with MRI image fusion.

VC13; Siemens Healthcare, Erlangen, Germany), and the IMRT plans for dosimetric comparison were generated using the Eclipse TPS (version V11; Varian Medical Systems, Palo Alto, CA, USA). The IMCT plans used for comparison with IMRT in the current study were those used for the re-RT for the participating patients.
The optimization constraints of the two types of plans were basically identical. The prescription doses ranged from 57 to $60 \mathrm{GyE}$ (at 2.5-3.0 GyE per daily fraction) for IMCT.

Ninty-five percent of the isodose line should cover the CTV for gross tumor and $90 \%$ dose line should 
cover the PTV for gross disease. The CTV for other subclinical diseases should be irradiated to $90 \%$ of the assigned dose level if applicable. All plans were reviewed from least three physicians, one dosimetrist and one senior physicist.

Recovery of the OARs from the first course of radiation was set at $70 \%$, and the dose constraints of the OARs followed the TD5/5 reported by Emami except for the optic nerve (D20 <30 GyE), brain stem (Dmax $<45$ GyE), spinal cord (Dmax $<30$ GyE), temporal lobes (V40 $<7.66 \mathrm{~cm}^{2}$; V50 $<4.66 \mathrm{~cm}^{2}$ ). ${ }^{18}$ And, it is strictly related to the previous already received dose.

\section{Comparisons of the treatment plans}

Comparison between the IMCT and IMRT plans focused on the dose coverage to the targets, as well as conformity and heterogeneity. The conformity index (CI), calculated from the standard formula used to determine conformity of the CTV, $\left[\left(\mathrm{CTV}^{2} 5^{2}\right) /\left(\mathrm{CTV}^{*} \mathrm{~V} 95\right)\right] .{ }^{19}$ The closer to 1 the value was, the better the conformity was. The homogeneity index (HI) was used as the standard formula to evaluate the homogeneity of the CTV, [(D2-D98)/D50]. ${ }^{20}$ The closer the HI value was to zero, the more homogeneous the plan was. For the CTV and all OARs, both the dose distributions and dosevolume histograms (DVHs) were assessed. A range of variables were assessed for each OAR patient and each plan, including mean dose (Dmean), minimum dose (Dmin), maximum dose (point and/or in a significant volume, Dmax), volume receiving a fixed-dose level (e.g., V30 is the volume receiving at least $30 \%$ of the prescribed dose) and dose levels delivered to a certain volume (e.g., D5).

\section{Statistical methodology}

All patients treated at SPHIC were included in a prospectively designed registry and database for future analyses. The clinical data of the 10 patients included in the study were analyzed using the SPSS 22.0 software. Measurement data were expressed as medians and quartiles (Q1, Q3), while the two types of planning dosimetry were compared using the matching Wilcoxon rank-sum test. $P<0.05$ was considered statistically significant.

\section{Results}

\section{Characteristics of patients and their}

\section{treatment}

This study included 10 patients with locally recurrent diseases treated with salvage IMCT. The recurrent disease was staged according to the 7th Edition of the American Joint Committee on Cancer (AJCC) staging classifications. The characteristics of the patient, their disease and treatment were detailed in Table 1 .

\section{Comparisons of dose coverage and distribution to the targets}

Both the IMCT and IMRT plans for all 10 patients covered the CTV adequately and met our requirements. Figure 2 demonstrates a typical comparison of the dose distribution by IMCT and IMRT planning in transverse, coronal, and sagittal sections. Approximately, $95 \%$ of the dose curve covers the target area and the prescription dose in the target coverage is basically identical. No significant difference was observed between the two techniques in dose coverage of the CTV.

The results of CTV coverage are shown in Table 2. All of the dose data acquired by this study were not normally distributed. Therefore, parameters were compared between the two systems using the matching Wilcoxon rank-sum test. There was no significant difference between the two systems in terms of the target area.

\section{DVH}

A typical DVH for both IMCT and IMRT plans is shown in Figure 3. The distribution of CTV in the IMCT plan was similar to the IMRT plan. The dose to the spinal cord, brain stem, and parotid gland in the IMCT plan was considerably lower than those in the IMRT plan. Furthermore, the dose to the TMJs, optical nerves, chiasm and temporal lobes in the IMCT plan was slightly lower than those in the IMRT plan.

\section{Dosimetric analyses of OARs}

Table 3 summarizes the quantitative comparison between the delivered IMCT plan and the reference IMRT plan for all OARs. The direction of recurrent tumors in NPC is not symmetrical; different cases will show differences in the distribution of parallel OARs on both the left and right sides. Therefore, in this study, we compared parallel OARs merged together.

\section{Discussion}

Historically, re-irradiation remains the most effective principle for patients with locally recurrent NPC. ${ }^{21}$ The reasons underlying the recurrence of NPC may be associated with the intrinsic radio-resistance of the primary tumor, 
Table I Patient characteristics

\begin{tabular}{|c|c|c|c|c|c|c|c|c|}
\hline Patient & Gender & Age & $\begin{array}{l}\text { Recurrent stage } \\
\text { (TNM) }\end{array}$ & $\begin{array}{l}\text { Induction } \\
\text { chemo }\end{array}$ & $\begin{array}{l}\text { Concurrent } \\
\text { chemo }\end{array}$ & $\begin{array}{l}\text { Total dose } \\
\text { (GyE) }\end{array}$ & Fraction & $\begin{array}{l}\text { Daily dose } \\
\text { (GyE) }\end{array}$ \\
\hline I & M & 68 & rT4NOM0, IVA & $\mathrm{TP} * 3$ & DDP & 57.5 & 23 & 2.5 \\
\hline 2 & $\mathrm{~F}$ & 38 & rT3NOMO, III & - & - & 57 & 19 & 3 \\
\hline 3 & $\mathrm{~F}$ & 42 & rT3NOMO, III & $\mathrm{TP} * 3$ & - & 57 & 19 & 3 \\
\hline 4 & $M$ & 46 & rTONIMO, II & TP*3 & - & 60 & 20 & 3 \\
\hline 5 & M & 34 & rT4NOM0, IVA & GP*3 & - & 60 & 24 & 2.5 \\
\hline 6 & $\mathrm{~F}$ & 52 & rT2NOMO, II & - & - & 60 & 20 & 3 \\
\hline 7 & M & 70 & rT4NOMO, IVA & TP*2 & - & 60 & 20 & 3 \\
\hline 8 & M & 33 & rT4NIM0, IVA & - & - & 60 & 20 & 3 \\
\hline 9 & M & 36 & rT4N2M0, IVA & GP*3 & - & 60 & 20 & 3 \\
\hline 10 & $M$ & 68 & rT3NOMO, III & - & - & 60 & 20 & 3 \\
\hline
\end{tabular}

Abbreviations: M, male; F, female; TNM, tumor, node, metastasis system; TP, docetaxel plus cisplatin; GP, gemcitabine plus cisplatin; DDP, cisplatin; GyE, Gray equivalent.

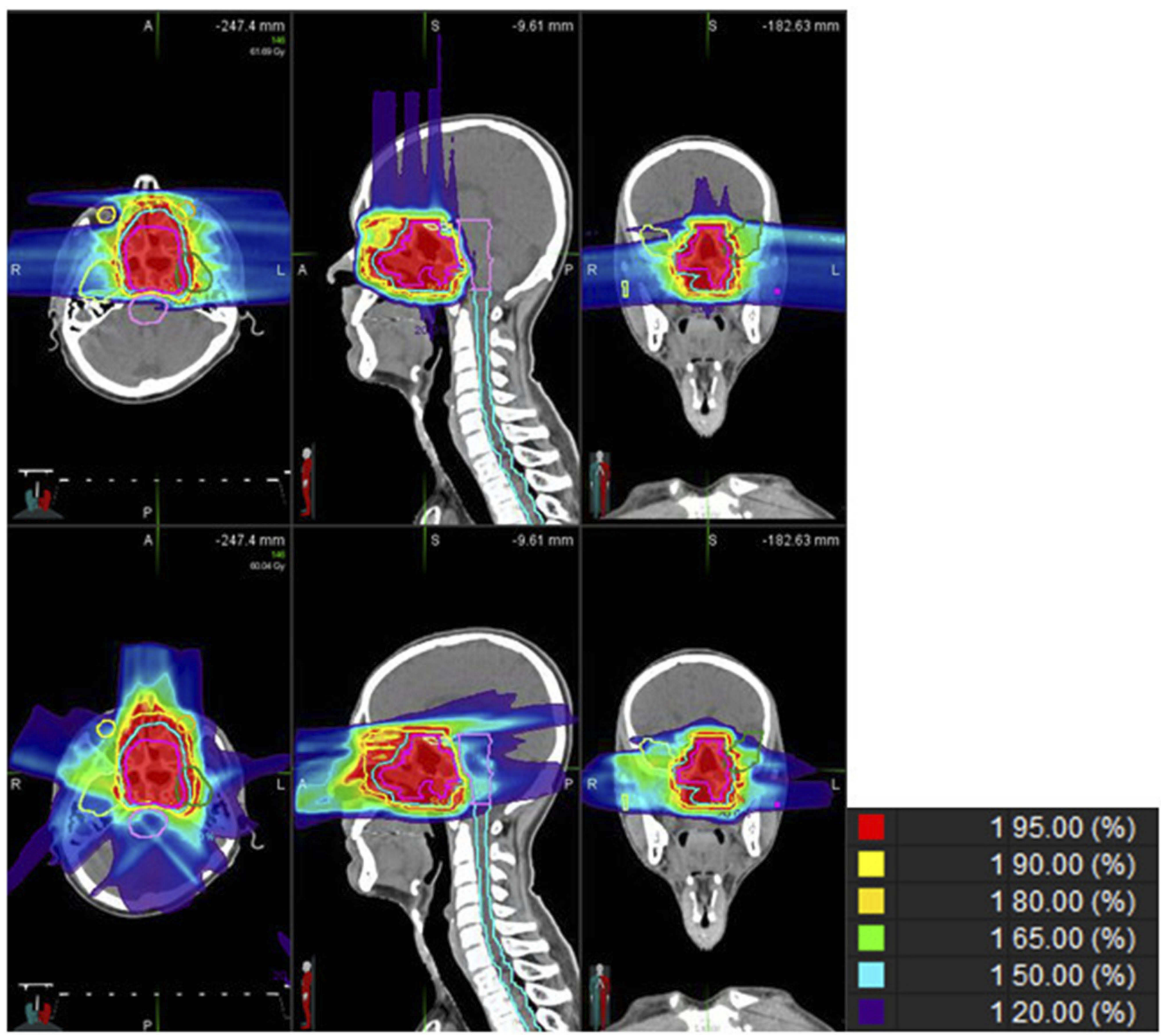

Figure 2 Dose distribution of intensity-modulated carbon-ion-based radiation therapy (IMCT) and intensity-modulated photon-based radiation therapy (IMRT) planning in one patient which demonstrates a typical comparison of the dose distribution by IMCT and IMRT planning in transverse, coronal, and sagittal sections. 
Table 2 Dosimetric summary, $\mathrm{Cl}$ and $\mathrm{HI}$ of target volumes

\begin{tabular}{|l|l|l|l|l|}
\hline & & IMCT & IMXT & P-value \\
\hline & DI & $61.810(60.577,62.620)$ & $62.375(61.710,62.715)$ & 0.203 \\
CTV & D2 & $61.380(59.992,62.287)$ & $62.240(61.600,62.557)$ & 0.092 \\
& D50 & $59.865(57.350,60.108)$ & $60.640(58.580,60.912)$ & 0.074 \\
& D95 & $57.655(55.253,58.705)$ & $58.775(56.762,59.410)$ & 0.203 \\
& D98 & $55.690(52.072,58.065)$ & $57.085(54.060,59.045)$ & 0.093 \\
& D99 & $54.270(46.197,57.698)$ & $54.925(51.070,58.795)$ & 0.203 \\
& V95 & $98.115(95.422,99.638)$ & $97.600(84.187,99.037)$ & 0.314 \\
& Cl & $0.963(0.911,0.990)$ & $0.971(0.920,0.990)$ & 0.314 \\
& HI & $0.084(0.058,0.146)$ & $0.089(0.054,0.137)$ & 0.285 \\
\hline
\end{tabular}

Abbreviations: CTV, clinical target volume; IMCT, intensity-modulated carbonion-based radiation therapy; IMXT, X-ray-based intensity-modulated radiation therapy; DI, dose levels delivered to $1 \%$ of volume; D2, dose levels delivered to $2 \%$ of volume; D50, dose levels delivered to half volume; D95, dose levels delivered to $95 \%$ of volume; D98, dose levels delivered to $98 \%$ of volume; D99, dose levels delivered to $99 \%$ of volume; V95, the volume receiving at least $95 \%$ of the prescribed dose; $\mathrm{Cl}$, conformity index; $\mathrm{HI}$, homogeneity index.

inadequate initial radiotherapy dose and the lack of initial treatment for certain sites. However, it is reported that 6$85 \%$ of patients with recurrent NPC after re-RT therapy will experience different types of complications, including dry mouth, hearing loss, ear effusion, brain necrosis and nasopharyngeal bleeding. ${ }^{22-26}$ The occurrence of these complications, especially the serious ones, mainly depends on the dose and the mode of radiotherapy. Unfortunately, local recurrence after high-dose photon RT may have a biological basis, as Kong and colleagues reported radioresistant cancer cells that survived the whole course of treatment in their study. ${ }^{27}$ Clearly, the initial IMRT can increase the difficulty of re-radiotherapy for recurrent NPC.

Particle therapy, such as proton-based or carbon-ionbased intensity-modulated radiotherapy (IMPT or IMCT) may represent a more favorable option. The physical advantage of particle therapy offers even more pronounced sparing of normal tissue. These include a sharp lateral penumbra and Bragg peak. When the beams enter the tumor, a large amount of energy is deposited with a short time window to form a narrow and pointed Bragg peak, and after that the energy is rapidly attenuated. Beam energy determines the depth of the Bragg peak. Thus, it is important that RT aims to protect the normal tissues of the head and neck region, particularly in those areas which have received radiotherapy previously. According to many research studies, particle therapy for primary or recurrent NPC would provide superior dose distributions. ${ }^{28,29}$ In addition to better physical properties, the carbon ion features a high LET ray with a significantly higher RBE than photons and protons. According to the tissue type and end point of the study, the RBE of the carbon atom can be
3 to 5. There are some reports showing that high LET radiation inflicts more damage by directly breaking DNA double-strands; these breaks are more difficult to repair. ${ }^{29}$ Thus, radiotherapy based on high LET radiation, such as IMCT, can improve clinical efficacy, especially for tumor cells with photon resistance properties.

Some dosimetry studies have reported that proton radiotherapy can reduce the dose to normal tissue around a tumor by approximately 50\% compared with conventional IMRT, thus reducing the incidence of adverse reactions in the normal tissues. Therefore, proton RT can safely increase the radiation dose for tumors, especially when a tumor is adjacent to critical organs. ${ }^{30}$ Carbon ion is not easily scattered and can produce a better distribution than a proton because of its larger mass. The dose distribution of the carbon ion at the edge of the field can decrease more rapidly with lateral distance and depth, so it is easier to target higher doses to a tumor which may lie adjacent to critical organs. Moreover, the Bragg peak of the carbon ion is narrower than for a proton, so we can conclude that the carbon ion might provide better protection of normal tissues. ${ }^{9}$

Carbon-ion radiotherapy has been used successfully in the treatment of chordoma/chondrosarcoma of the skull base, melanoma and adenoid cystic carcinoma of the head and neck. ${ }^{10-14}$ Clinical trials of IMCT have been conducted at the Heidelberg Ion-Beam Therapy Center (HIT) in Germany, the National Institute of Radiological Sciences (NIRS) in Japan and SPHIC. In addition, IMCT has been used in these institutions as a routine treatment for cancer patients. The safety of OARs, including the optic nerve, optic chiasm, spinal cord and brain stem has also been reported. 
A

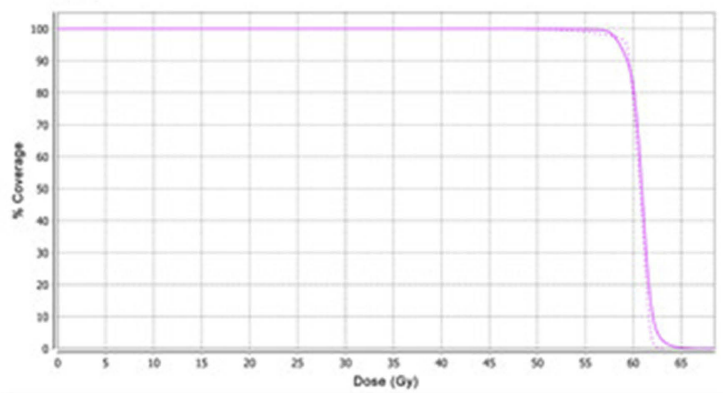

B

C
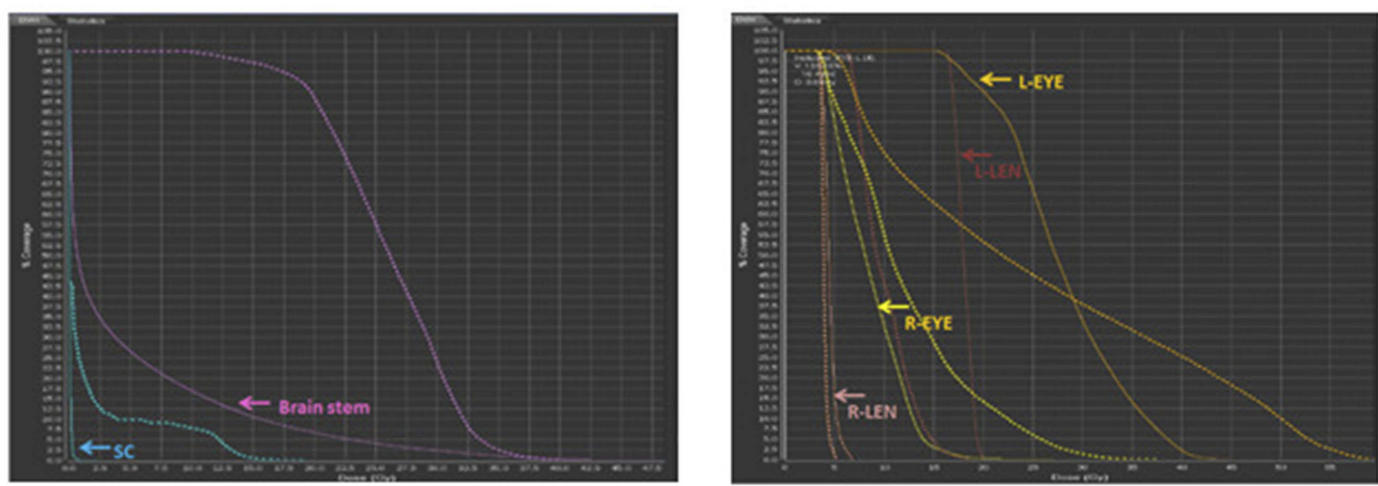

D

E
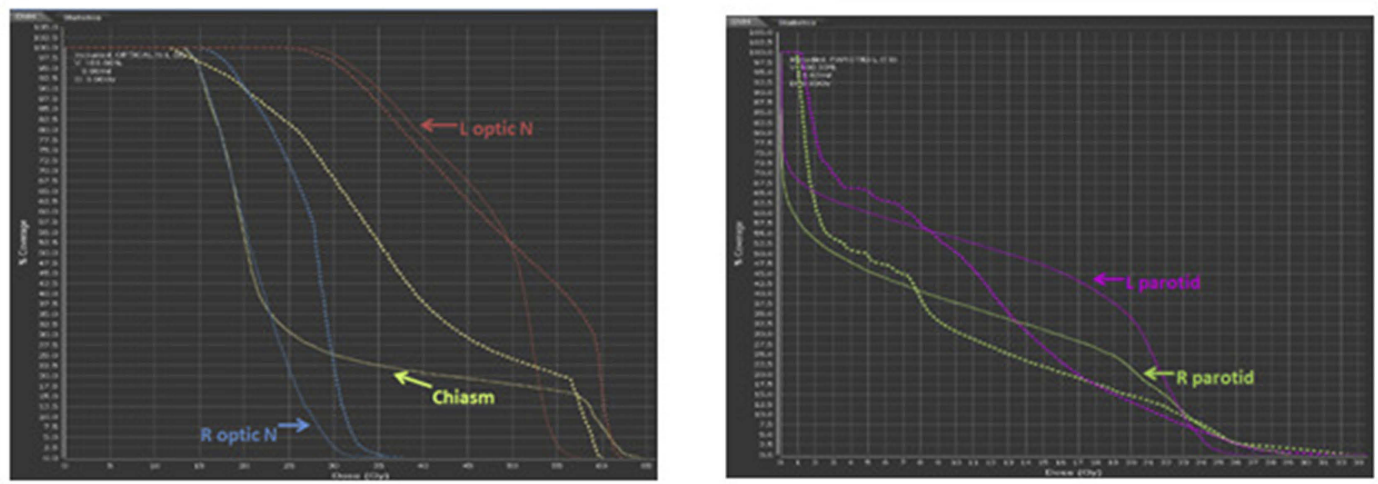

F

G
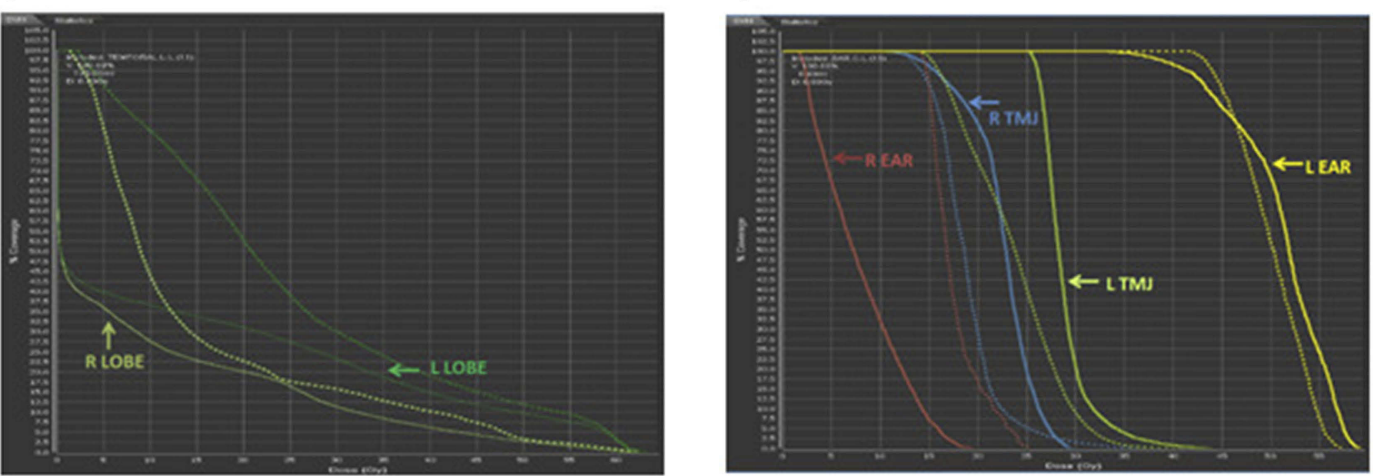

Figure 3 Cumulative dose-volume histograms for clinical target volume (CTV) and organs at risk (OARs) for the same representative patient. The solid line is intensitymodulated carbon ion radiation therapy (IMCT) plan and the dotted line is intensity-modulated radiation therapy (IMRT) plan. (A) Cumulative dose-volume histograms (DVH) for CTV for the same representative patient; (B) cumulative DVH for brain stem and spinal cord for the same representative patient; (C) cumulative DVH for lens and eyeball for the same representative patient; (D) cumulative DVH for optic nerve and chiasm for the same representative patient; (E) cumulative DVH for parotid gland for the same representative patient; (F) cumulative DVH for temporal lobe for the same representative patient; and (G) cumulative DVH for temporomandibular joint and inner ear for the same representative patient. 
Table 3 Dosimetric comparison of OARs between treated IMCT plans and reference IMRT plans

\begin{tabular}{|c|c|c|c|c|}
\hline & & IMCT & IMXT & $P$-value \\
\hline \multirow[t]{3}{*}{ Spinal cord } & DI & $3.425(0.547,1 \mid .242)$ & $14.640(12.692,16.065)$ & 0.005 \\
\hline & Dmean & $0.365(0.172,1.347)$ & $1.970(1.385,2.830)$ & 0.005 \\
\hline & Dmax & $9.365(2.100,18.055)$ & $19.655(17.175,22.140)$ & 0.022 \\
\hline \multirow[t]{3}{*}{ Brain stem } & DI & $25.375(18.510,30.162)$ & $36.090(29.382,39.772)$ & 0.005 \\
\hline & Dmean & $4.325(2.255,6.745)$ & $22.050(13.477,24.330)$ & 0.005 \\
\hline & Dmax & $35.680(28.455,37.177)$ & $41.460(33.222,43.600)$ & 0.022 \\
\hline \multirow[t]{2}{*}{ Parotid glands } & V30 & $0.000(0.000,1.565)$ & $2.580(0.237,9.482)$ & 0.028 \\
\hline & Dmean & $9.610(5.710,16.925)$ & $8.505(4.160,15.630)$ & 0.263 \\
\hline \multirow[t]{3}{*}{ Chiasm } & DI & $25.210(22.137,41.027)$ & $33.530(26.430,47.940)$ & 0.005 \\
\hline & D5 & $28.190(25.545,36.307)$ & $33.940(14.522,44.607)$ & 0.799 \\
\hline & Dmax & $32.520(29.935,4 I .170)$ & $37.640(15.035,47.525)$ & 0.959 \\
\hline \multirow[t]{3}{*}{ Optic nerves } & DI & $32.660(21.155,29.200)$ & $33.110(12.637,38.257)$ & 0.627 \\
\hline & D5 & $26.135(19.310,29.635)$ & $31.750(11.865,36.855)$ & 0.156 \\
\hline & Dmax & $30.680(21.710,36.762)$ & $34.640(13.095,45.045)$ & 0.575 \\
\hline \multirow[t]{3}{*}{ Eyes } & Dmax & II.090 (0.090, 29.880) & 35.275 (1I.692, 4I.997) & $<0.001$ \\
\hline & Dmean & $0.630(0.000,7.357)$ & $6.455(2.452,7.527)$ & 0.006 \\
\hline & D5 & $3.750(0.000,19.385)$ & $22.665(6.397,29.200)$ & 0.001 \\
\hline \multirow[t]{3}{*}{ Lens } & Dmax & $0.105(0.000,1.307)$ & $3.140(1.590,4.817)$ & 0.003 \\
\hline & Dmean & $0.010(0.000,0.230)$ & $2.250(1.265,2.667)$ & 0.002 \\
\hline & D5 & $0.040(0.000,0.657)$ & $2.980(1.532,3.907)$ & 0.004 \\
\hline \multirow[t]{3}{*}{ Temporal lobes } & DI & $47.950(36.287,56.375)$ & $55.910(38.497,59.257)$ & 0.005 \\
\hline & Dmean & $7.150(4.945,9.977)$ & $12.765(7.872,17.965)$ & 0.003 \\
\hline & Dmax & $55.03(49.852,61.607)$ & $61.200(54.082,62.432)$ & 0.022 \\
\hline \multirow[t]{2}{*}{ TMJs } & DI & $39.245(26.825,47.952)$ & $38.540(30.252,50.197)$ & 0.179 \\
\hline & Dmax & $42.470(28.047,50.562)$ & $42.775(32.900,51.230)$ & 0.163 \\
\hline \multirow[t]{2}{*}{ Inner ears } & D5 & $37.610(18.162,44.677)$ & $42.120(31.142,50.100)$ & 0.001 \\
\hline & Dmean & $25.990(12.195,33.937)$ & $36.425(26.725,45.525)$ & $<0.001$ \\
\hline
\end{tabular}

Abbreviations: IMCT, intensity-modulated carbon-ion-based radiation therapy; IMXT, X-ray-based intensity-modulated radiation therapy; DI, dose levels delivered to I\% of volume; Dmean, mean dose; Dmax, maximum dose (point and/or in a significant volume); D5, dose levels delivered to $5 \%$ of volume; V30, the volume receiving at least $30 \%$ of the prescribed dose; TMJs, temporomandibular joints.

Our present study suggests that both IMCT and IMRT plans can meet the requirements for target volume dose coverage. In addition, there was no statistically significant difference in terms of treatment target dose coverage when compared between the two plans. From a quantitative point of view, there were no significant differences in D1, D2, D50, D95, D98 and D99 of CTV, when comparing between IMCT and IMRT planning. However, the V95 was slightly improved in the IMCT plan than in the IMRT plan (IMCT vs IMRT: 98.115 vs $97.600, P>0.05$ ), although such difference was not statistically significant. Such finding might be caused by small sample size of our study; however, given the small magnitude of the difference in dose, no clinical difference is expected.
Furthermore, according to the CI, it is possible that the IMCT technique may generate a better degree of confinement for high-dose levels around target volumes. The number of subfields in the design of a treatment plan implies that the plan can be adjusted further so that the target area becomes more conformable. There were no statistical differences between the two groups in terms of CI (IMCT vs IMRT: 0.963 vs $0.971, P>0.05$ ), meaning that there was no difference in conformity; this may be related to the physical advantages of the carbon ion. Another important issue is that homogeneity still needs to be explored between the two plans. In the current study, the dose distribution at the target was almost the same when compared between IMCT and IMRT plans (IMCT vs 
IMRT: 0.084 vs $0.089, P>0.05)$. However, it must be emphasized that only two parallel-opposed fields were used in the IMCT plan, and the CI may be further improved with more beam directions, although such improvement may not be clinically relevant. On the contrary, 5-9 fixed fields in the IMRT plan were used.

In addition to killing tumor cells as efficiently as possible, radiotherapy also should reduce normal tissues damage around the target area as much as possible. The structure of the organs around a NPC is very specific, as the anatomy of the head and neck area is incredibly complex; consequently, it is very challenging to coordinate the destruction of tumors in this area and protect normal tissues. Comparing the two plans, it is clear that the IMCT guarantees more sparing of select non-target tissue while both plans maintain sufficient coverage of the target. Firstly, protection afforded by IMCT to the spinal cord (Dmax of IMCT vs IMRT: 9.365 vs $19.655, P<0.05$ ) and brain stem (Dmax of IMCT vs IMRT: 35.680 vs 41.460 , $P<0.05)$ was significantly superior to that afforded by IMRT. For patients with recurrent NPC who have received radiotherapy during first treatment, protection of the spinal cord and brain stem is particularly imperative. It was also evident that the amount of irradiation to the spinal cord and brain stem in the IMCT plan was significantly lower. Consequently, IMCT is safer for patients with recurrent lesions close to the brain stem than IMRT.

Secondly, the protection of the parotid gland (V30 of IMCT vs IMRT: 0.000 vs $2.580, P<0.05$ ), chiasm (D1 of IMCT vs IMRT: 25.210 vs $33.530, P<0.05$ ), eyeball (Dmax of IMCT vs IMRT: 11.090 vs $35.275, P<0.05$ ), lens (Dmax of IMCT vs IMRT: 0.105 vs $3.140, P<0.05$ ), temporal lobe (Dmean of IMCT vs IMRT: 7.150 vs 12.765, $P<0.05$ ) and inner ear (D5 of IMCT vs IMRT: 37.610 vs $42.120, P<0.05)$ was significantly better than that of IMRT. The dose of the temporal lobe in the IMCT plan was significantly reduced, which is of great clinical significance for the reduction of complications such as radiation brain injury and brain necrosis because all patients received radiotherapy twice. Similarly, a smaller dose to the parotid gland may also reduce the risk of complications, such as dry mouth.

Thirdly, there was no statistically significant difference in the protection given to the optic nerve (Dmax IMCT vs IMRT: 30.680 vs $34.640, P>0.05$ ) and TMJ (Dmax IMCT vs IMRT: 42.470 vs $42.775, P>0.05)$ when compared between the IMCT and IMRT plans. This may be related to more patients with a late-stage classification; in our 10 patients, recurrent tumors were larger and closer. As a result, the IMCT plan failed to show any difference in protecting the optic nerve and TMJ. In this study, the IMCT plan was superior to the IMRT plan in terms of the level of protection given to the chiasm, eyeball, lens and parotid gland. We consider that if the sample number increases, the IMCT plan may also reduce the dose to the optic nerve and TMJ, which will alleviate the difficulty of mouth opening and reduce the chance of radioactive mandibular necrosis.

This study of dosimetric results might not be representative across a wider population of patients with different types of tumors, although it still provides a theoretical insight that the carbon ion improves the therapeutic ratio. To our knowledge, this is the first study to provide a detailed and comprehensive analysis of the role of IMCT over IMRT for locally recurrent NPC. There was no evident difference between the two plans in terms of target dose distribution and the target area covered. However, the protection provided by IMCT for some critical organs was significantly superior to that of IMRT, which would be very useful for long-term survival and the prognosis of patients with recurrent NPC.

Several pitfalls and limitations need to be considered for the present study. First, most of our recurrent NPC patients presented with large tumor loads and advanced stages, thus the tumors were adjacent to the OARs. In addition, the sample size of this study was relatively small. However, as the patients were selected to cover various scenarios for planning comparison, and the purpose of the study is not to compare the clinical outcome after two radiation technology, we consider our finding is valid to present a theoretical concept for the dosimetry advantage of IMCT over IMRT. Further investigation is needed to assess the clinical implication of IMCT.

\section{Conclusion}

Dosimetric comparisons of plan level between IMCT and IMRT show that the IMCT plan has the same target coverage, homogeneity and conformability as the IMRT plan. Furthermore, IMCT reduces the level of irradiation at the OARs. Compared to IMRT, IMCT can spare OARs in a more efficient manner while achieving the same dose to target coverage. This means that further improvement can be expected for normal tissue protection by the continued use of carbon-ion radiotherapy. Our present data, therefore, deserve further investigation. 


\section{Statement of ethical standards}

The Institution Review Board (IRB) of SPHIC approval was obtained for this trial, and it was performed in accordance with the ethical standards laid down in the 1964 Declaration of Helsinki and its later amendments.

\section{Disclosure}

Dr Lei Wang reports grants from Joint Breakthrough Project for New Frontier Technologies of the Shanghai Hospital Development Center (Project No. SHDC 12015118) and grants from Science and Technology Commission of Shanghai Municipality (Project Nos. 15411950102 and 15411950106), during the conduct of the study. The authors report no other conflicts of interest in this work.

\section{References}

1. Lee AW, Ma BB, Ng WT, et al. Management of nasopharyngeal carcinoma: current practice and future perspective. J Clin Oncol. 2015;33(29):3356-3364. doi:10.1200/JCO.2015.60.9347

2. Kong L, Lu JJ. Reirradiation of locally recurrent nasopharyngeal cancer: history, advances, and promises for the future. Chin Clin Oncol. 2016;5(2):26. doi:10.21037/cco

3. Wei WI, Kwong DL. Recurrent nasopharyngeal carcinoma: surgical salvage vs. additional chemoradiation. Curr Opin Otolaryngol Head Neck Surg. 2011;19(2):82-86. doi:10.1097/MOO.0b013e328344a599

4. Chua DT, Wei WI, Sham JS, Hung KN, Au GKH. Stereotactic radiosurgery versus gold grain implantation in salvaging local failures of nasopharyngeal carcinoma. Int J Radiat Oncol Biol Phys. 2007;69(2):469-474. doi:10.1016/j.ijrobp.2007.03.012

5. Han F, Zhao C, Huang SM, et al. Long-term outcomes and prognostic factors of re-irradiation for locally recurrent nasopharyngeal carcinoma using intensity-modulated radiotherapy. Clin Oncol ( $R$ Coll Radiol). 2012;24(8):569-576. doi:10.1016/j.clon.2011.11.010

6. Hua YJ, Han F, Lu LX, et al. Long-term treatment outcome of recurrent nasopharyngeal carcinoma treated with salvage intensity modulated radiotherapy. Eur $J$ Cancer. 2012;48(18):3422-3428. doi:10.1016/j.ejca.2012.06.016

7. Qiu S, Lin S, Tham IW, et al. Intensity-modulated radiation therapy in the salvage of locally recurrent nasopharyngeal carcinoma. Int J Radiat Oncol Biol Phys. 2012;83(2):676-683. doi:10.1016/j.ijrobp.2011.07.006

8. Pryzant RM, Wendt CD, Delclos L, Peters LJ. Re-treatment of nasopharyngeal carcinoma in 53 patients. Int $J$ Radiat Oncol Biol Phys. 1992;22(5):941-947. doi:10.1016/0360-3016(92)90792-g

9. Suit H, DeLaney T, Goldberg S, et al. Proton vs carbon ion beams in the definitive radiation treatment of cancer patients. Radiother Oncol. 2010;95(1):3-22. doi:10.1016/j.radonc.2010.01.015

10. Holliday EB, Kocak-Uzel E, Feng L, et al. Dosimetric advantages of intensity-modulated proton therapy for oropharyngeal cancer compared with intensity-modulated radiation: a case-matched control analysis. Med Dosim. 2016;41(3):189-194. doi:10.1016/j. meddos.2016.01.002

11. Schulz-Ertner D, Nikoghosyan A, Didinger B, et al. Therapy strategies for locally advanced adenoid cystic carcinomas using modern radiation therapy techniques. Cancer. 2005;104(2):338344. doi: $10.1002 / \mathrm{cncr} .21158$

12. Schulz-Ertner D, Nikoghosyan A, Hof H, et al. Carbon ion radiotherapy of skull base chondrosarcomas. Int J Radiat Oncol Biol Phys. 2007;67(1):171-177. doi:10.1016/j.jjrobp.2006.08.027
13. Schulz-Ertner D, Karger CP, Feuerhake A, et al. Effectiveness of carbon ion radiotherapy in the treatment of skull-base chordomas. Int $J$ Radiat Oncol Biol Phys. 2007;68(2):449-457. doi:10.1016/j.ijrobp.2006.12.059

14. Combs SE, Nikoghosyan A, Jaekel O, et al. Carbon ion radiotherapy for pediatric patients and young adults treated for tumors of the skull base. Cancer. 2009;115(6):1348-1355. doi:10.1002/cncr.24153

15. Kong L, Gao J, Hu J, et al. Phase I/II trial evaluating concurrent carbon-ion radiotherapy plus chemotherapy for salvage treatment of locally recurrent nasopharyngeal carcinoma. Chin J Cancer. 2016;35 (1):101. doi:10.1186/s40880-016-0164-5

16. Hodapp N. [The ICRU Report 83: prescribing, recording and reporting photon-beam intensity-modulated radiation therapy (IMRT)]. Strahlenther Onkol. 2012;188(1):97-99. doi:10.1007/s00066-011-0015-x

17. Sun Y, Yu XL, Luo W, et al. Recommendation for a contouring method and atlas of organs at risk in nasopharyngeal carcinoma patients receiving intensity-modulated radiotherapy. Radiother Oncol. 2014;110(3):390-397. doi:10.1016/j.radonc.2013.10.035

18. Hu J, Bao C, Gao J, et al. Salvage treatment using carbon ion radiation in patients with locoregionally recurrent nasopharyngeal carcinoma: initial results. Cancer. 2018;124(11):2427-2437. doi:10.1002/cncr.31318

19. Swanson EL, Indelicato DJ, Louis D, et al. Comparison of threedimensional (3D) conformal proton radiotherapy (RT), 3D conformal photon RT, and intensity-modulated RT for retroperitoneal and intraabdominal sarcomas. Int J Radiat Oncol Biol Phys. 2012;83(5):1549_ 1557. doi:10.1016/j.ijrobp.2011.10.014

20. Special considerations regarding absorbed-dose and dose-volume prescribing and reporting in IMRT. J ICRU. 2010;10(1):27-40. doi: $10.1093 / \mathrm{jicru} / \mathrm{ndq} 008$

21. Wei WI, Chan JY, Ng RW, et al. Surgical salvage of persistent or recurrent nasopharyngeal carcinoma with maxillary swing approach critical appraisal after 2 decades. Head Neck. 2011;33(7):969-975. doi:10.1002/hed.21558

22. Lee AW, Law SC, Foo W, et al. Retrospective analysis of patients with nasopharyngeal carcinoma treated during 1976-1985: survival after local recurrence. Int J Radiat Oncol Biol Phys. 1993;26(5):773782. doi:10.1016/0360-3016(93)90491-d

23. Wang CC. Re-irradiation of recurrent nasopharyngeal carcinoma treatment techniques and results. Int $J$ Radiat Oncol Biol Phys. 1987;13(7):953-956. doi:10.1016/0360-3016(87)90030-7

24. Yan JH, Hu YH, Gu XZ. Radiation therapy of recurrent nasopharyngeal carcinoma. Report on 219 patients. Acta Radiol Oncol. 1983;22(1):23-28.

25. Teo PM, Kwan WH, Chan AT, et al. How successful is high-dose (> or $=60 \mathrm{~Gy}$ ) reirradiation using mainly external beams in salvaging local failures of nasopharyngeal carcinoma? Int J Radiat Oncol Biol Phys. 1998;40(4):897-913.

26. Chang JT, See LC, Liao CT, et al. Locally recurrent nasopharyngeal carcinoma. Radiother Oncol. 2000;54(2):135-142.

27. Kong L, Wang L, Shen C, Hu C, Wang L, Lu JJ. Salvage intensitymodulated radiation therapy (IMRT) for locally recurrent nasopharyngeal cancer after definitive IMRT: a novel scenario of the modern era. Sci Rep. 2016;6:32883. doi:10.1038/srep32883

28. Feehan PE, Castro JR, Phillips TL, et al. Recurrent locally advanced nasopharyngeal carcinoma treated with heavy charged particle irradiation. Int $J$ Radiat Oncol Biol Phys. 1992;23(4):881-884. doi:10.1016/0360-3016(92)90663-3

29. Lin R, Slater JD, Yonemoto LT, et al. Nasopharyngeal carcinoma: repeat treatment with conformal proton therapy-dose-volume histogram analysis. Radiology. 1999;213(2):489-494. doi:10.1148/radiology.213.2.r99nv29489

30. Nichols RC Jr, Huh SN, Prado KL, et al. Protons offer reduced normal-tissue exposure for patients receiving postoperative radiotherapy for resected pancreatic head cancer. Int J Radiat Oncol Biol Phys. 2012;83(1):158-163. doi:10.1016/j.ijrobp.2011.05.045 


\section{Publish your work in this journal}

Cancer Management and Research is an international, peer-reviewed open access journal focusing on cancer research and the optimal use of preventative and integrated treatment interventions to achieve improved outcomes, enhanced survival and quality of life for the cancer patient.
The manuscript management system is completely online and includes a very quick and fair peer-review system, which is all easy to use. Visit http://www.dovepress.com/testimonials.php to read real quotes from published authors.

Submit your manuscript here: https://www.dovepress.com/cancer-management-and-research-journal 